\title{
Analysis of flow of outer meshing elliptic gear pump based on Fluent
}

\author{
Qingdun Zeng ${ }^{\mathrm{a}}$ and Junzhi Sun \\ School of Civil Engineering and Transportation, South China University of Technology, Guangzhou, 510640, China
}

\begin{abstract}
The output flow is one of the important indicators of gear pump performance. External meshing elliptic gear pump is a new type of displacement pump, whose performance is better than circular gear pump, especially in terms of output flow. The influence of rotating speed, centrifugal rate and number of teeth of an elliptical gear pump on the flow of the gear pump was analyzed by using the dynamic mesh technique in the software Fluent ${ }^{\circledR}$. The results show that the greater the revolving speed, the greater the output flow rate is; the greater the centrifugal rate, the greater the output flow is; and the effect of the number of teeth on the output flow can be neglected.
\end{abstract}

Keywords: elliptical gear pump; flow; fluent; dynamic mesh.

\section{Introduction}

With the rapid development of computer and numerical control technology, circular gear pumps have been widely used. A large number of scholars have studied them extensively. For example, papers [14] have studied them from turbulent kinetic energy, dissipation rate, cavitation, tooth profile curve equation and noise, respectively, which can improve the working performance of circular gear pumps. Thus it can be seen that the research and development of gear pumps with good performance is very significant. However, these studies mainly are based on the circle pitch curve, and the research results can not fundamentally improve the inherent performance of gear pumps, such as output flow [5]. Therefore, in this paper, a new type of displacement pump-elliptic gear pump is numerically simulated. Its structural diagram is shown in Fig. 1. Due to the complexity of internal flow field in elliptical gear pump, the transient simulation of the internal flow field of elliptical gear pump can be realized easily by using the moving grid technique. So, the present paper makes a numerical simulation on the internal flow field of elliptical gear pump based on the software Fluent ${ }^{\circledR}$, which provides a reference for design and optimization of structures and new gear pumps.

\section{Theoretical analysis of fluid dynamics for elliptical gear pump}

Although boundary conditions and internal flow field of elliptic gear pump are complex, the basic control equations of fluid are still to be satisfied, such as the continuity equation and the NavierStokes equation. Their tensor expressions [6] are as follows:

\footnotetext{
${ }^{\mathrm{a}}$ Corresponding author : emqdzeng@scut.edu.cn
} 


$$
\begin{aligned}
& \frac{\partial \rho}{\partial t}+\frac{\partial\left(\rho u_{i}\right)}{\partial x_{i}}=0 \\
& \frac{\partial\left(\rho u_{i}\right)}{\partial t}=-\frac{\partial \prod_{i k}}{\partial x_{k}}
\end{aligned}
$$

For incompressible fluids, the expression of $\prod_{i k}$ is:

$$
\prod_{i k}=-p \delta_{i k}+\eta\left(\frac{\partial u_{i}}{\partial x_{k}}+\frac{\partial u_{k}}{\partial x_{i}}\right)
$$

where $p$ is pressure and $\eta$ is the dynamic viscosity.

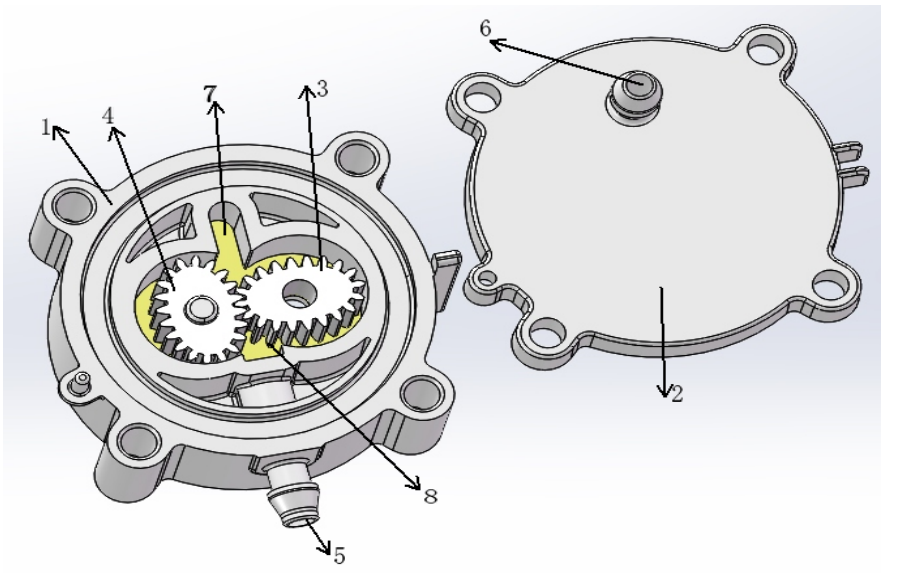

Figure 1. Structural diagram of elliptical gear pump.

1-Base; 2-Upper; 3-Driving gear; 4-Driven gear; 5-Inlet; 6-Outlet; 7-Discharge chamber; 8-Suction chamber.

When the gear pump works, the internal flow field generates turbulence. The fluid closed to the wall rotates with the gears, and the standard model of turbulence has been unable to accurately reflect the flow conditions. The turbulence model of RNG (RNG is short of renormalization group) $\kappa-\varepsilon$ [4] not only revises turbulent viscosity, but also considers the rotating flow pattern in an average flow, where $\kappa$ is the turbulent kinetic energy and $\varepsilon$ is the turbulent dissipation rate. It can better simulate the flow field of pump work. The expressions of the turbulence model of RNG $\kappa-\varepsilon$ [4] are as follows:

$$
\begin{aligned}
\frac{\partial(\rho \kappa)}{\partial t}+\frac{\partial}{\partial x_{i}}\left(\rho \kappa u_{i}\right)= & \frac{\partial}{\partial x_{j}}\left(\alpha_{\kappa} \mu_{e f f} \frac{\partial \kappa}{\partial x_{j}}\right)+G_{k}+G_{b}-\rho \varepsilon-Y_{M}+S_{k} \\
\frac{\partial(\rho \varepsilon)}{\partial t}+\frac{\partial}{\partial x_{i}}\left(\rho \varepsilon u_{i}\right)= & \frac{\partial}{\partial x_{j}}\left(\alpha_{\varepsilon} \mu_{e f f} \frac{\partial \varepsilon}{\partial x_{j}}\right)+C_{1 \varepsilon} \frac{\varepsilon}{\kappa}\left(G_{k}+C_{3 \varepsilon} G_{b}\right) \\
& -C_{2 \varepsilon} \rho \frac{\varepsilon^{2}}{\kappa}-R_{\varepsilon}+S_{\varepsilon}
\end{aligned}
$$

where $G_{k}$ represents the generation of turbulence kinetic energy due to the mean velocity gradients, $G_{b}$ is the turbulence kinetic energy generated by average velocity gradient, $Y_{M}$ indicates the contribution of the fluctuating dilatation in compressible turbulence to the overall dissipation rate, 
$\alpha_{\kappa}$ and $\alpha_{\varepsilon}$ respectively are the effective Prandtl constants corresponding to $\kappa$ and $\varepsilon, S_{\kappa}$ and $S_{\varepsilon}$ respectively are the user-defined source terms, and $C_{1 \varepsilon}, C_{2 \varepsilon}$ and $C_{3 \varepsilon}$ are constants.

\section{Computing model of dynamic grid}

Dynamic grid technique can be used to simulate the boundary of rigid body rotation or boundary deformation caused by the flow field shape changing over time. The technology provides three models respectively: layering, spring smoothing and local re-meshing. Because the triangular grids are used in the paper, two model of spring smoothing and local re-meshing are employed to update the grids when gears rotate. The general formula of dynamic grid computation model is as follows:

$$
\frac{\mathrm{d}}{\mathrm{d} t} \int_{V} \rho \phi \mathrm{d} V+\int_{\partial V} \rho \phi\left(\vec{u}-\vec{u}_{s}\right) \mathrm{d} \vec{A}=\int_{\partial V} \Gamma \nabla \phi \mathrm{d} \vec{A}+\int_{V} S_{\phi} \mathrm{d} V
$$

where $\vec{u}_{s}$ is the velocity vector of liquid and $\vec{u}_{s}$ is the deforming velocity of moving mesh, $\Gamma$ is the diffusion coefficient, $S_{\phi}$ is the source term of flux $\phi$, and $\partial V$ is the volume boundary.

\section{Calculation model and setting of solution conditions}

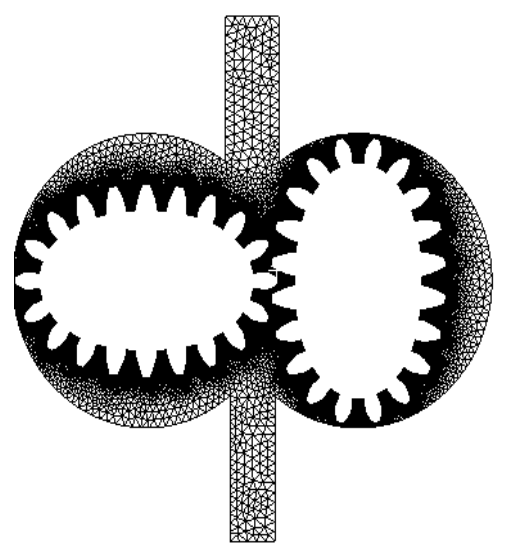

Figure 2. Meshing of 2-D model of external meshing ellipse gear pump.

According to the actual sizes of a certain type of elliptical gear pump, a 3-D model of internal flow field is drawn by the software SolidWorks ${ }^{\circledR}$. Due to the symmetry of the model, the 3-D model is simplified as a 2-D model in order to reduce the computation time. The software Workbench ${ }^{\circledR}$ is used to divide meshes, the mesh is a triangle, the number of nodes is 75684, and the number of elements is 140750. The generated meshes are shown in Fig.2.

The pitch curves of two gears in Fig. 2 are the two-order ellipses with the same geometry, which can be satisfied with the unidirectional continuous transmission. The parametric expression of the pitch curve of the left gear is as follows:

$$
r_{1}=\frac{p_{1}}{1-e \cos (2 \psi)}, p_{1}=a\left(1-e^{2}\right)
$$

The transmission relationbetween two gears is shown in Fig. 3 [7]. In Fig. 3, the left is the driving gear, while the right is the driven gear. When the left gear rotates $\psi$ over counterclockwise, the right gear rotates $\theta$ over clockwise. The center distance of two rotating shafts is: 


$$
r_{1}+r_{2}=2 a
$$

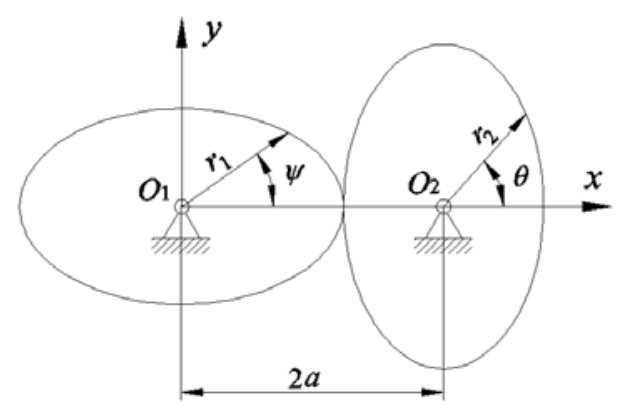

Figure 3. Relationship diagram of elliptic gear transmission.

The expression of rotation speed of the driven gear can be derived from the relationship of transmission ratio and Eqs. (6) and (7), which is

$$
\dot{\theta}=\dot{\psi} \frac{1-e^{2}}{1-2 e \cos (2 \psi)+e^{2}}
$$

where $\dot{\psi}$ and $\dot{\theta}$ are the rotation speeds of driving and driven gears, respectively, $e$ is the centrifugal rate, and $a$ is the semi-major axis.

When the ellipse gear pump works, the complexity of boundary conditions and the rotation can produce a turbulent flow, so we choose the model of RNG $\kappa-\varepsilon$ [8]. The working medium is water, whose physical properties are the density of $998.2 \mathrm{~kg} / \mathrm{m}^{3}$ and the viscosity of $0.001003 \mathrm{~kg} / \mathrm{ms}$, respectively. The inlet boundary condition is the pressure inlet. The inlet pressure is $101325 \mathrm{~Pa}$ on the basis of operating conditions. The outlet boundary condition is the pressure outlet. The outlet pressure is $0 \mathrm{~Pa}$ based on operating conditions. The standard atmospheric pressure is selected as the pressure value at the center of the outlet, which is the operating conditions. We choose "PISO" (Pressure Implicit with Splitting of Operators) as the calculation method of solver. For the spatial discretization format, the selection of gradient is the least square method based on the control volume, the pressure set is "PRESTO!" (a kind of pressure discretization scheme) method, and the rest is two-order upwind scheme. The solution control is by default. The rotation speed of two gears is set by UDF (UserDefined-Function), which is:

\#include"udf.h"

\#include"math.h"

real $\mathrm{A}=0.0$;

DEFINE_CG_MOTION(Right,dt, vel, omega,time,dtime)

$$
\{
$$

$\mathrm{A}=94.24778 *$ time;

omega[2]=-94.24778*(1-pow $(0.25,2)) /(1-2 * 0.25 * \cos (2 * A)+\operatorname{pow}(0.25,2))$; \}

DEFINE_CG_MOTION(Left,dt, vel, omega,time,dtime)

$$
\{
$$

omega[2]=94.24778;

\} 


\section{Results and discussions of simulation}

Under the condition of the same pressure angle $\left(20^{\circ}\right)$, the output flow rate of elliptic gear pump is analyzed from three aspects: the rotation speed, the centrifugal rate and the number of teeth. Specific calculation results are listed in Tables 1 to 3.

The results from Table 1 show that as the speed increases, the output flow increases gradually, but the tendency of flow increase lowers. Table 2 shows that the change of the number of teeth has little effect on the output flow. Table 3 shows that as the increase of the centrifugal rate, the output flow increases, but the tendency of flow increase lowers. Tables 2 and 3 show that although the elliptical gear pump is a displacement pump, the cause of volume change is different from the circle gear pump, depending on the area that the long and short axes sweep the area in the unit time. Therefore, compared to the centrifugal rate, the effect of the number of teeth on the output flow can be ignored.

Table 1. Output flow at different speeds

\begin{tabular}{|c|c|c|c|c|c|c|}
\hline $\begin{array}{c}\text { Rotation } \\
\text { speed (rpm) }\end{array}$ & $\begin{array}{c}\text { Teeth } \\
\text { number }\end{array}$ & $\begin{array}{c}\text { Modulus } \\
(\mathrm{mm})\end{array}$ & $\begin{array}{c}\text { Centrifug } \\
\text { al rate }\end{array}$ & $\begin{array}{c}\text { Semi-major } \\
\text { axis }(\mathrm{mm})\end{array}$ & $\begin{array}{c}\text { Flow } \\
(\mathrm{kg} / \mathrm{s})\end{array}$ & $\begin{array}{c}\text { Growth } \\
\text { range }\end{array}$ \\
\hline 900 & 20 & 0.3843 & 0.22 & 3.755 & 3.7 & \\
\hline 1500 & 20 & 0.3843 & 0.22 & 3.755 & 4.416 & $19.35 \%$ \\
\hline 2000 & 20 & 0.3843 & 0.22 & 3.755 & 4.735 & $7.2 \%$ \\
\hline
\end{tabular}

Table 2. Output flow of different teethes

\begin{tabular}{|c|c|c|c|c|c|c|}
\hline $\begin{array}{c}\text { Rotation speed } \\
(\mathrm{rpm})\end{array}$ & $\begin{array}{c}\text { Teeth } \\
\text { number }\end{array}$ & $\begin{array}{c}\text { Modulus } \\
(\mathrm{mm})\end{array}$ & $\begin{array}{c}\text { Centrifugal } \\
\text { rate }\end{array}$ & $\begin{array}{c}\text { Semi-major } \\
\text { axis }(\mathrm{mm})\end{array}$ & $\begin{array}{c}\text { Flow } \\
(\mathrm{kg} / \mathrm{s})\end{array}$ & $\begin{array}{c}\text { Growth } \\
\text { range }\end{array}$ \\
\hline 900 & 20 & 0.3843 & 0.22 & 3.755 & 3.7 & \\
\hline 900 & 28 & 0.2798 & 0.22 & 3.828 & 3.84 & $3.78 \%$ \\
\hline 900 & 40 & 0.199 & 0.22 & 3.889 & 3.35 & $-1.28 \%$ \\
\hline
\end{tabular}

Table 3. Output flow under different centrifugal rates

\begin{tabular}{|c|c|c|c|c|c|c|}
\hline $\begin{array}{c}\text { Rotation speed } \\
(\mathrm{rpm})\end{array}$ & $\begin{array}{c}\text { Teeth } \\
\text { number }\end{array}$ & $\begin{array}{c}\text { Modulus } \\
(\mathrm{mm})\end{array}$ & $\begin{array}{c}\text { Centrifugal } \\
\text { rate }\end{array}$ & $\begin{array}{c}\text { Semi-major } \\
\text { axis }(\mathrm{mm})\end{array}$ & $\begin{array}{c}\text { Flow } \\
(\mathrm{kg} / \mathrm{s})\end{array}$ & $\begin{array}{c}\text { Growth } \\
\text { range }\end{array}$ \\
\hline 900 & 20 & 0.3843 & 0.22 & 3.755 & 3.7 & \\
\hline 900 & 20 & 0.377 & 0.25 & 3.66 & 4.73 & $27.84 \%$ \\
\hline 900 & 20 & 0.371 & 0.28 & 3.578 & 5.018 & $6.1 \%$ \\
\hline
\end{tabular}

\section{Summary}

In this paper, the output flow of elliptic gear pump is studied from three aspects: the rotational speed, the number of teeth and the centrifugal rate. The results show that the effect of the number of teeth on the output flow is negligible, compared with the rotational speed and the centrifugal rate. Therefore, only if the number of teeth meets the minimum number of teeth that the processing requires, the output flow of the elliptic gear pump is determined by the rotational speed and the centrifugal rate.

\section{References}

1. N. Ertürk, A. Vernet, Small-scale characteristics and turbulent statistics of the flow in an external gear pump by time-resolved PIV, Flow Meas. Inst. 29, 52 (2013).

2. J. Zhou, A. Vacca, P. Casoli, A novel approach for predicting the operation of external gear pumps under cavitating conditions, Simulation Modelling Practice \& Theory 45, 35 (2014).

3. J. D. Zhao, Analysis of profile of gear tooth for internal gear pump, Machinery Design \& Manufacture (2), 83 (2003). (in Chinese)

4. L. Wu and L. C. Gu, Analysis of internal flow field of external gear pump based on Fluent, Coal 
Mine Machinery 36(10), 145 (2015). (in Chinese)

5. M. Y. Li, M. Zhang, J. Sun, Analysis of flow characteristics gear rotator pump, Journal of Shanghai University of Engineering Science 23(1), 6 (2009). (in Chinese)

6. Landau, Lifshitz (eds.), Fluid Dynamics (Higher Education Press, 2013). (in Chinese)

7. Z. B. Lu, CAD/CAM of ellipse gear, Master's thesis, Sichuan University (2006). (in Chinese)

8. F. J. Wang (ed.), Computational Fluid Dynamics Analysis (Tsinghua University Press, 2004). (in Chinese) 